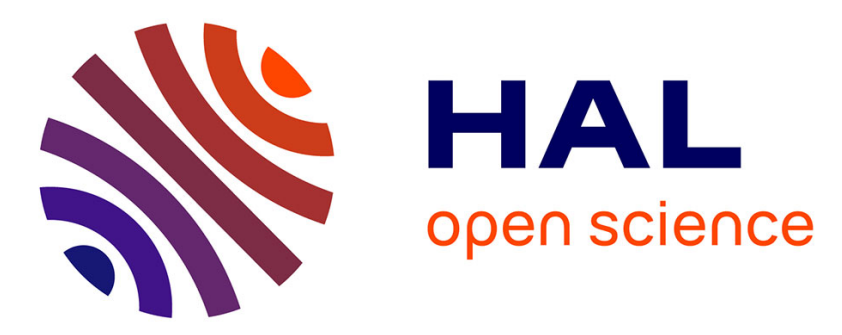

\title{
Representation theorems and fundamental solutions for micropolar solid-fluid mixtures under steady state vibrations
}

Ionel-Dumitrel Ghiba

\section{- To cite this version:}

Ionel-Dumitrel Ghiba. Representation theorems and fundamental solutions for micropolar solid-fluid mixtures under steady state vibrations. European Journal of Mechanics - A/Solids, 2010, 29 (6), pp.1034. 10.1016/j.euromechsol.2010.07.002 . hal-00687353

\section{HAL Id: hal-00687353 https://hal.science/hal-00687353}

Submitted on 13 Apr 2012

HAL is a multi-disciplinary open access archive for the deposit and dissemination of scientific research documents, whether they are published or not. The documents may come from teaching and research institutions in France or abroad, or from public or private research centers.
L'archive ouverte pluridisciplinaire HAL, est destinée au dépôt et à la diffusion de documents scientifiques de niveau recherche, publiés ou non, émanant des établissements d'enseignement et de recherche français ou étrangers, des laboratoires publics ou privés. 


\section{Accepted Manuscript}

Title: Representation theorems and fundamental solutions for micropolar solid-fluid mixtures under steady state vibrations

Authors: Ionel-Dumitrel Ghiba

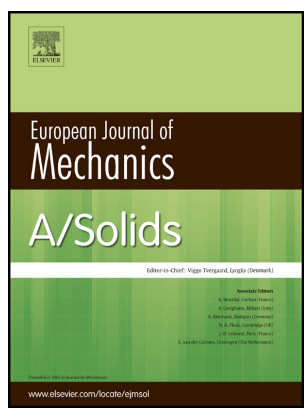

PII: $\quad$ S0997-7538(10)00093-8

DOI: $\quad$ 10.1016/j.euromechsol.2010.07.002

Reference: EJMSOL 2628

To appear in: European Journal of Mechanics / A Solids

Received Date: 2 April 2009

Revised Date: 30 June 2010

Accepted Date: 5 July 2010

Please cite this article as: Ghiba, I.-D. Representation theorems and fundamental solutions for micropolar solid-fluid mixtures under steady state vibrations, European Journal of Mechanics / A Solids (2010), doi: 10.1016/j.euromechsol.2010.07.002

This is a PDF file of an unedited manuscript that has been accepted for publication. As a service to our customers we are providing this early version of the manuscript. The manuscript will undergo copyediting, typesetting, and review of the resulting proof before it is published in its final form. Please note that during the production process errors may be discovered which could affect the content, and all legal disclaimers that apply to the journal pertain. 
Elsevier Editorial System(tm) for European Journal of Mechanics - A/Solids Manuscript Draft

Manuscript Number: EJMSOL-D-09-00065R2

Title: Representation theorems and fundamental solutions for micropolar solid-fluid mixtures under steady state vibrations

Article Type: Article

Keywords: microstructure; solid-fluid interaction; fundamental solution

Corresponding Author: researcher Ionel-Dumitrel I. Ghiba, M.D.

Corresponding Author's Institution: "Octav Mayer" Mathematics Institute, Romanian Academy of

First Author: Ionel-Dumitrel I. Ghiba, M.D.

Order of Authors: Ionel-Dumitrel I. Ghiba, M.D.

Abstract: The isothermal theory of binary micropolar solid-fluid mixture is considered in this paper. For the dynamical problem, a Galerkin type representation of the solution is established. Then, a fundamental solution is given for the three dimensional partial differential system which describes the steady vibrations. Also, some basic properties of the fundamental solution and a direct application to the point load problem are presented. 


\title{
Representation theorems and fundamental solutions for micropolar solid-fluid mixtures under steady state vibrations
}

\author{
Ionel-Dumitrel Ghiba \\ "Octav Mayer" Mathematics Institute, Romanian Academy of Science, Iaşi Branch, \\ Blvd. Carol I, no. 8, 700506 - Iaşi, Romania $\mathcal{E}$ \\ Faculty of Mathematics, Al. I. Cuza University of Iaşi, \\ Blvd. Carol I, no. 11, 700506 - Iaşi, Romania
}

\begin{abstract}
The isothermal theory of binary micropolar solid-fluid mixture is considered in this paper. For the dynamical problem, a Galerkin type representation of the solution is established. Then, a fundamental solution is given for the three dimensional partial differential system which describes the steady vibrations. Also, some basic properties of the fundamental solution and a direct application to the point load problem are presented.
\end{abstract}

Keywords: microstructure, solid-fluid interaction, steady state vibrations, fundamental solution

\section{Introduction}

A microcontinuum media, roughly speaking, is a continuum media whose properties and behaviour are affected by the local motions and deformations of the primitive elements. A special case of microcontinuum media is that of micropolar continua. In the micropolar continuum theory, the rotational degrees of freedom play a central role. Thus, we have six degrees of freedom, instead of three degrees of freedom considered in classical elasticity and fluid mechanics. The number of published works in these fields presently exceeds several hundred papers. A modern presentation, the state-of-art and the intended applications of these theories can be found in the books (Eringen, 1999, 2001). The higher-order or higher-grade continuum theories are necessary to capture size effects in small length scales, where the fundamental assumption of the classical continuum theory is that the (physical, chemical,

Email address: ghiba_dumitrel@yahoo.com (Ionel-Dumitrel Ghiba) 
mechanical, etc.) properties of a material are uniformly distributed throughout its volume fails (Tekoglu \& Onck, 2008). We remind that the theory of micropolar elastic materials has many applications concerning cellular solids (Lakes, 1983, 1986; Mora \& Wass, 2000).

On the other hand, many natural or synthetic materials are not pure materials. They are mixtures of two or more co-existence constituents. Sometimes the presence of a constituent can be ignored, if there is a preponderant constituent, but in many situations the local mechanical effects of each ingredient of the mixture cannot be ignored. In classical continuum theory (Rajagopal \& Tao, 1995), a mixture is idealized by assuming that every point in the mixture is occupied simultaneously by each constituent. A mixture is thereby envisioned as a superposition of several continuous media.

Taking into account the microstructural motions Twiss \& Eringen (1971, 1972 ) introduce the mixture theory of materials with microstructure. In the last years many papers (Eringen, 2003; Ieşan, 2007, 2009; Chiriţă \& Galeş, 2008) got back in discussion the study of mixtures with microstructure. Eringen (2003) has developed a continuum theory for a mixture of a micropolar elastic solid and a micropolar viscous fluid. This theory can be successfully applied to the study of engineering materials, as well as soils, rocks, granular materials, sand and underground water mixtures. Consolidation problems in the building industry, earthquake problems, oil exploration problems and cellular solids can be studied with the help of the mixture theories (de Boer, 2005; Elangovan et al., 2008).

In the framework of the theory developed by Eringen (2003), the existence, uniqueness, continuous data dependence of the solution of the initialboundary value problem, the asymptotic partition of the energy and the stability problem have been studied by Ghiba (2006, 2007, 2008, 2009).

In various boundary-value problems from continuum mechanics it is important to give a representation of the general solution of the field equations in term of elementary (harmonic, biharmonic etc.) functions and to find the fundamental solution. In the micropolar elasticity, this type of results have been established by Ieşan (1971), Şandru (1966, 1975), Dragoş (1980, 1983). Ramkissoon \& Majundar (1976 a,b) obtained the fundamental solution for slow, steady motion of micropolar fluids, in planar and three dimensional space. Olmstead \& Majumdar (1983) gave the fundamental solution for a Oseen flow in the two dimensional case. These results have been completed by the study by Shu \& Lee (2008).

In the present paper we consider the isothermal theory of a binary homogeneous mixture of an isotropic micropolar elastic solid with an incompressible micropolar viscous fluid. In the second section the basic equations of this theory are presented. In the third section, using the method introduced 
by Moisil (1952), we establish a representation of Galerkin type for the dynamical problem. In the next section we use some Galerkin representations in order to determine the fundamental solutions for the three-dimensional problem governing the motion of a micropolar solid-fluid mixture in the case of steady state vibrations. A direct application to the point load problem is presented in the last section of the paper.

The representations of the solution and the fundamental solutions for micropolar mixtures have not been studied yet. The equations of the theory developed by Eringen (2003) combine the usual linearized system of equations for isotropic micropolar elastic solids with those for incompressible micropolar viscous fluid and become a complex system of partial differential equations. We outline that the fundamental solutions of the static problems and of the steady oscillations problem for other types of mixtures are constructed in (Svanadze, 1993, 1996; Ciarletta, 1995; Ieşan, 1996; de Boer \& Svanadze, 2004; Galeş, 2004 a,b; Svanadze \& de Boer, 2005). We also note that in the theories of pure materials with microstructure the proposed problems have been studied by Ieşan \& Pompei (1995); Ieşan \& Nappa (2001), Svanadze et al. (2007), Nappa (2008) and Cialetta et al. (2009).

\section{Basic equations}

The space under consideration is the Euclidean three-dimensional space. We refer the motion of a continuum to a fixed system of rectangular Cartesian axes $O x_{k}(k=1,2,3)$. The Latin subscripts, unless otherwise specified, are understood to range over the integers 1,2,3, the Greek subscripts are confined to the range 1,2 and superscripts $\sigma=s, f$ denote the micropolar elastic solid and the incompressible micropolar fluid, respectively. Summation over repeated subscripts and other typical conventions for differential operations are implied such as comma followed by a subscript to denote the partial derivative with respect to the corresponding cartesian coordinate.

We suppose that the mixture is chemical inert, and the fluid is incompressible.

In this paper we use the following notations:

- $\rho^{\sigma}$ - the density of the $\sigma$ th constituent;

- $\pi^{f}$ - the dynamic pressure in the fluid species;

- $u_{r}^{\sigma}$ - the displacement of the $\sigma$ th constituent;

- $\phi_{r}^{\sigma}$ - the microrotation vector of the $\sigma$ th constituent;

- $v_{r}^{\sigma}$ - the velocity of the $\sigma$ th constituent; 
- $\nu_{r}^{\sigma}$ - the microrotation rate of the $\sigma$ th constituent;

- $\lambda^{s}, \mu^{s}, k^{s}, \alpha^{s}, \beta^{s}, \gamma^{s}$ - the micropolar elastic constants for micropolar elastic solid;

- $\mu^{f}, k^{f}, \alpha^{f}, \beta^{f}, \gamma^{f}$ - the micropolar fluid viscosities;

- $\xi$ - the momentum generation coefficient due to the velocity difference;

- $\varpi-$ the momentum generation coefficient due to the difference in gyration;

- $F_{r}^{\sigma}$ - the body force;

- $L_{r}^{\sigma}$ - the body couple;

- $j^{\sigma}-$ the microinertia density.

The field equations of the linear theory of isothermal micropolar solid-fluid mixtures are (Eringen, 2003)

$$
\begin{gathered}
\left(\lambda^{s}+\mu^{s}\right) u_{j, r j}^{s}+\left(\mu^{s}+k^{s}\right) u_{r, j j}^{s}+k^{s} \varepsilon_{r j k} \phi_{k, j}^{s}- \\
-\xi\left(\frac{\partial}{\partial t} u_{r}^{s}-v_{r}^{f}\right)+F_{r}^{s}=\rho^{s} \frac{\partial^{2}}{\partial t^{2}} u_{r}^{s}, \\
\left(\alpha^{s}+\beta^{s}\right) \phi_{j, r j}^{s}+\gamma^{s} \phi_{r, j j}^{s}+k^{s}\left(\varepsilon_{r j k} u_{k, j}^{s}-2 \phi_{r}^{s}\right)- \\
-\varpi\left(\frac{\partial}{\partial t} \phi_{r}^{s}-\nu_{r}^{f}\right)+L_{r}^{s}=\rho^{s} j^{s} \frac{\partial^{2}}{\partial t^{2}} \phi_{r}^{s}, \\
-\pi_{r, r}^{f}+\left(\mu^{f}+k^{f}\right) v_{r, j j}^{f}+k^{f} \varepsilon_{r j k} \nu_{k, j}^{f}+\xi\left(\frac{\partial}{\partial t} u_{r}^{s}-v_{r}^{f}\right)+F_{r}^{f}=\rho^{f} \frac{\partial}{\partial t} v_{r}^{f}, \\
\left(\alpha^{f}+\beta^{f}\right) \nu_{j, r j}^{f}+\gamma^{f} \nu_{r, j j}^{f}+k^{f}\left(\varepsilon_{r j k} v_{k, j}^{f}-2 \nu_{r}^{f}\right)+ \\
+\varpi\left(\frac{\partial}{\partial t} \phi_{r}^{s}-\nu_{r}^{f}\right)+L_{r}^{f}=\rho^{f} j^{f} \frac{\partial}{\partial t} \nu_{r}^{f}, \\
v_{r, r}^{f}=0
\end{gathered}
$$

where $\varepsilon_{r j k}$ is the permutation symbol. It is easy to see that if we ignore the microstructure then the above equations will describe the behaviour of mixtures which have as constituents an isotropic elastic solid and a classical fluid with $\lambda^{s}, \mu^{s}$ and $\mu^{f}$ the classical Lamé constants.

In this paper we suppose that the dissipation potential and the internal energy density (Eringen, 2003) are positive definite. This is true if and only if

$$
\begin{aligned}
& 3 \lambda^{s}+2 \mu^{s}+k^{s}>0, \quad 2 \mu^{\sigma}+k^{\sigma}>0, \quad k^{\sigma}>0, \varpi>0, \quad \xi>0, \\
& 3 \alpha^{\sigma}+\beta^{\sigma}+\gamma^{\sigma}>0, \quad \gamma^{\sigma}+\beta^{\sigma}>0, \quad \gamma^{\sigma}-\beta^{\sigma}>0, \quad(\sigma=s, f) .
\end{aligned}
$$




\section{Complete solutions of the field equations}

In this section we establish a Galerkin type representation of the solution of the field equations.

Let us introduce the differential operators:

$$
\begin{aligned}
Q_{1}^{s}(\Delta) & =\left(\mu^{s}+k^{s}\right) \Delta-\xi \frac{\partial}{\partial t}-\rho^{s} \frac{\partial^{2}}{\partial t^{2}}, \quad Q_{2}^{s}(\Delta)=\gamma^{s} \Delta-2 k^{s}-\varpi \frac{\partial}{\partial t}-\rho^{s} j^{s} \frac{\partial^{2}}{\partial t^{2}} \\
Q_{3}^{s}(\Delta) & =\left(\lambda^{s}+\mu^{s}\right) \Delta+Q_{1}^{s}, \quad Q_{4}^{s}(\Delta)=\left(\alpha^{s}+\beta^{s}\right) \Delta+Q_{2}^{s}, \\
Q_{5}^{s}(\Delta) & =\left(k^{s}\right)^{2} \Delta+Q_{1}^{s} Q_{2}^{s}, \\
Q_{1}^{f}(\Delta) & =\left(\mu^{f}+k^{f}\right) \Delta-\xi-\rho^{f} \frac{\partial}{\partial t}, \quad Q_{2}^{f}(\Delta)=\gamma^{f} \Delta-2 k^{f}-\varpi-\rho^{f} j^{f} \frac{\partial}{\partial t} \\
Q_{3}^{f}(\Delta) & =\left(\alpha^{f}+\beta^{f}\right) \Delta+Q_{2}^{f}, \quad Q_{4}^{f}(\Delta)=\left(k^{f}\right)^{2} \Delta+Q_{1}^{f} Q_{2}^{f}, \\
P_{1}(\Delta) & =\varpi^{2} \frac{\partial}{\partial t}-Q_{2}^{f} Q_{2}^{s}, \quad P_{2}(\Delta)=\xi^{2} \frac{\partial}{\partial t}-Q_{1}^{f} Q_{1}^{s},
\end{aligned}
$$

where $\Delta=\frac{\partial^{2}}{\partial x_{1}^{2}}+\frac{\partial^{2}}{\partial x_{2}^{2}}+\frac{\partial^{2}}{\partial x_{3}^{2}}$ is the Laplace operator.

In the above quantities and in the following, if $Q_{n}, n=1,2, \ldots, m$ are differential operators and $G$ is, for example, a $C^{\infty}(\mathbb{R})$ function, we use the notation

$$
Q_{1} Q_{2} \ldots Q_{m} G \equiv Q_{1}\left(Q_{2}\left(\ldots\left(Q_{m}(G)\right) \ldots\right)\right) .
$$

With the help of these operators, we also define the following differential operators:

$$
\begin{aligned}
& D_{1}(\Delta)=-\varpi^{2} \frac{\partial}{\partial t}+Q_{3}^{f} Q_{4}^{s} \\
& D_{2}(\Delta)=Q_{5}^{s} Q_{4}^{f}+2 \xi \varpi k^{s} k^{f} \Delta \frac{\partial}{\partial t}-\left(\varpi^{2} Q_{1}^{s} Q_{1}^{f}+\xi^{2} Q_{2}^{s} Q_{2}^{f}\right) \frac{\partial}{\partial t}+\xi^{2} \varpi^{2} \frac{\partial^{2}}{\partial t^{2}} \\
& D_{3}(\Delta)=D_{2}-\left(Q_{4}^{f} Q_{2}^{s}-\frac{\partial}{\partial t} \varpi^{2} Q_{1}^{f}\right) Q_{3}^{s}, \\
& D_{4}(\Delta)=Q_{4}^{f} Q_{3}^{f}\left[\left(k^{s}\right)^{2}-\left(\alpha^{s}+\beta^{s}\right) Q_{1}^{s}\right]+2 \xi \varpi k^{s} k^{f} Q_{3}^{f} \frac{\partial}{\partial t}+ \\
& +\varpi^{2} Q_{1}^{s}\left[\left(k^{f}\right)^{2}-\left(\alpha^{f}+\beta^{f}\right) Q_{1}^{f}\right] \frac{\partial}{\partial t}+\xi^{2}\left(\alpha^{s}+\beta^{s}\right) Q_{2}^{f} Q_{3}^{f} \frac{\partial}{\partial t}+\left(\alpha^{f}+\beta^{f}\right) \xi^{2} \varpi^{2} \frac{\partial^{2}}{\partial t^{2}} \\
& D_{5}(\Delta)=Q_{5} \varpi\left(k^{f}\right)^{2}+\xi k^{s} k^{f}\left(\varpi^{2} \frac{\partial}{\partial t}+Q_{3}^{f} Q_{4}^{s}\right)+\varpi\left\{\left[\left(\alpha^{s}+\beta^{s}\right) Q_{2}^{f}+\right.\right. \\
& \left.\left.+\left(\alpha^{f}+\beta^{f}\right) Q_{2}^{s}+\left(\alpha^{f}+\beta^{f}\right)\left(\alpha^{s}+\beta^{s}\right) \Delta\right] P_{2}+\left(k^{s}\right)^{2} Q_{1}^{f} Q_{2}^{f}\right\} \\
& D_{6}(\Delta)=Q_{5}^{s} Q_{4}^{s}\left[\left(k^{f}\right)^{2}-\left(\alpha^{f}+\beta^{f}\right) Q_{1}^{f}\right]+2 \xi \varpi k^{s} k^{f} Q_{4}^{s} \frac{\partial}{\partial t}+ \\
& +\varpi^{2} Q_{1}^{f}\left[\left(k^{s}\right)^{2}-\left(\alpha^{s}+\beta^{s}\right) Q_{1}^{s}\right] \frac{\partial}{\partial t}+\xi^{2}\left(\alpha^{f}+\beta^{f}\right) Q_{2}^{s} Q_{4}^{s} \frac{\partial}{\partial t}+\xi^{2} \varpi^{2}\left(\alpha^{s}+\beta^{s}\right) \frac{\partial^{2}}{\partial t^{2}}
\end{aligned}
$$


Using the method introduced by Moisil (1952) we construct a representation of Galerkin type for the solution of the dynamical problem.

Theorem 3.1. Let

$$
\begin{aligned}
& u_{r}^{s}=\Delta Q_{3}^{s} D_{1}\left(-\varpi^{2} \frac{\partial}{\partial t} Q_{1}^{f}+Q_{4}^{f} Q_{2}^{s}\right) G_{r}^{s}+D_{1} D_{3} G_{j, r j}^{s}+ \\
& +\varepsilon_{r j k} \Delta Q_{3}^{s} D_{1}\left(k^{s} Q_{4}^{f}+\xi \varpi k^{f} \frac{\partial}{\partial t}\right) H_{j, k}^{s}+ \\
& +\Delta Q_{3}^{s} D_{1}\left(\varpi k^{s} k^{f} \Delta+\xi P_{1}\right) G_{r}^{f}-Q_{3}^{s} D_{1}\left(\varpi k^{s} k^{f} \Delta+\xi P_{1}\right) G_{j, r j}^{f}- \\
& -\varepsilon_{i j k} \Delta Q_{3}^{s} D_{1}\left(\varpi k^{s} Q_{1}^{f}+\xi k^{f} Q_{2}^{s}\right) H_{j, k}^{f}-\xi P_{, r} \text {, } \\
& \phi_{r}^{s}=\varepsilon_{r j k} \Delta Q_{3}^{s} D_{1}\left(k^{s} Q_{4}^{f}+\xi \varpi k^{f} \frac{\partial}{\partial t}\right) G_{j, k}^{s}+ \\
& +\Delta Q_{3}^{s} D_{1}\left[\left(k^{f}\right)^{2} \Delta Q_{1}^{s}-P_{2} Q_{2}^{f}\right] H_{r}^{s}+\Delta Q_{3}^{s} D_{4} H_{j, r j}^{s}- \\
& -\varepsilon_{r j k} D_{1} \Delta Q_{3}^{s}\left(\varpi k^{f} Q_{1}^{s}+\xi k^{s} Q_{2}^{f}\right) G_{j, k}^{f}+ \\
& +\Delta Q_{3}^{s} D_{1}\left(\xi k^{s} k^{f} \Delta+\varpi P_{2}\right) H_{r}^{f}-\Delta Q_{3}^{s} D_{5} H_{j, r j}^{f}, \\
& v_{r}^{f}=\frac{\partial}{\partial t} \Delta Q_{3}^{s} D_{1}\left(\varpi k^{s} k^{f} \Delta+\xi P_{1}\right) G_{r}^{s}-\frac{\partial}{\partial t} Q_{3}^{s} D_{1}\left(\varpi k^{s} k^{f} \Delta+\xi P_{1}\right) G_{j, r j}^{s}- \\
& -\varepsilon_{r j k} \frac{\partial}{\partial t} \Delta Q_{3}^{s} D_{1}\left(\varpi k^{f} Q_{1}^{s}+\xi k^{s} Q_{2}^{f}\right) H_{j, k}^{s}+ \\
& +\Delta Q_{3}^{s} D_{1}\left(-\varpi^{2} \frac{\partial}{\partial t} Q_{1}^{s}+Q_{5}^{s} Q_{2}^{f}\right) G_{r}^{f}-Q_{3}^{s} D_{1}\left(-\varpi^{2} \frac{\partial}{\partial t} Q_{1}^{s}+Q_{5}^{s} Q_{2}^{f}\right) G_{j, r j}^{f}+ \\
& +\varepsilon_{r j k} \Delta Q_{3}^{s} D_{1}\left(\xi \varpi k^{s} \frac{\partial}{\partial t}+k^{f} Q_{5}^{s}\right) H_{j, k}^{f}+Q_{3}^{s} P_{, r}, \\
& \nu_{r}^{f}=-\varepsilon_{r j k} \Delta \frac{\partial}{\partial t} Q_{3}^{s} D_{1}\left(\varpi k^{s} Q_{1}^{f}+\xi k^{f} Q_{2}^{s}\right) G_{j, k}^{s}+ \\
& +\frac{\partial}{\partial t} \Delta Q_{3}^{s} D_{1}\left(\xi k^{s} k^{f} \Delta+\varpi P_{2}\right) H_{r}^{s}-\frac{\partial}{\partial t} \Delta Q_{3}^{s} D_{5} H_{j, r j}^{s}+ \\
& +\varepsilon_{r j k} \Delta Q_{3}^{s} D_{1}\left(\xi \varpi k^{s} \frac{\partial}{\partial t}+k^{f} Q_{5}^{s}\right) G_{j, k}^{f}+ \\
& +\Delta Q_{3}^{s} D_{1}\left[\left(k^{s}\right)^{2} \Delta Q_{1}^{f}-P_{2} Q_{2}^{s}\right] H_{r}^{f}+\Delta Q_{3}^{s} D_{6} H_{j, r j}^{f}, \\
& \pi^{f}=\xi \frac{\partial}{\partial t} D_{1} D_{2} G_{r, r}^{s}-Q_{3}^{s} D_{1} D_{2} G_{r, r}^{f}-\left(\xi^{2} \frac{\partial}{\partial t}-Q_{1}^{f} Q_{3}^{s}\right) P,
\end{aligned}
$$

where $G_{r}^{s}, G_{r}^{f}, H_{r}^{s}, F_{r}^{f}$ and $P$ satisfy

$\Delta Q_{3}^{s} D_{1} D_{2} G_{r}^{\sigma}=-F_{r}^{\sigma}, \Delta Q_{3}^{s} D_{1} D_{2} H_{r}^{\sigma}=-L_{r}^{\sigma}, \Delta Q_{3}^{s} P=0(\sigma=s, f)$.

Then $u_{r}^{s}, v_{r}^{f}, \phi_{r}^{s}, \nu_{r}^{f}$ and $\pi^{f}$ satisfy the equations (2.1).

Proof. It is easy to see that for every $\mathbf{A} \in C^{3}\left(\mathbb{R}^{3}\right) \times C^{3}\left(\mathbb{R}^{3}\right) \times C^{3}\left(\mathbb{R}^{3}\right)$ we have

$$
\varepsilon_{r j k} A_{j, k i}=0, \quad \varepsilon_{j m n} A_{m, n r j}=0, \quad \varepsilon_{r j k} A_{m, k m j}=0
$$


and

$$
\varepsilon_{k r j} \varepsilon_{k m n}=\delta_{r m} \delta_{j n}-\delta_{r n} \delta_{j m} .
$$

To prove that $v_{r}^{f}$ satisfies the incompressibility condition $(2.1)_{5}$, we use the relations (3.4), (3.6) and the relations (3.5) for $\mathbf{A}=\mathbf{H}^{s}$ and $\mathbf{A}=\mathbf{H}^{f}$.

By a direct substitution of the relations (3.3) in (2.1) and using the relations (3.4), (3.5), (3.6) and the identities

$$
\begin{aligned}
Q_{3}^{s} D_{4}-\varpi \frac{\partial}{\partial t} D_{5} & =D_{1}\left\{k^{s}\left(k^{s} Q_{4}^{f}+\xi \varpi k^{f} \frac{\partial}{\partial t}\right)-\left(\alpha^{s}+\beta^{s}\right)\left[\left(k^{f}\right)^{2} \Delta Q_{1}^{s}-P_{2} Q_{2}^{f}\right]\right\}, \\
Q_{3}^{s} D_{5}-\varpi D_{6} & =D_{1}\left[k^{s}\left(\varpi k^{s} Q_{1}^{f}+\xi k^{f} Q_{2}^{s}\right)+\left(\alpha^{s}+\beta^{s}\right)\left(\xi k^{f} k^{s} \Delta+\varpi P_{2}\right)\right], \\
Q_{3}^{f} D_{5}-\varpi D_{4} & =D_{1}\left[\left(\alpha^{f}+\beta^{f}\right)\left(\xi k^{f} k^{s} \Delta+\varpi P_{1}\right)+k^{f}\left(\varpi k^{f} Q_{1}^{s}+\xi k^{s} Q_{2}^{f}\right)\right], \\
Q_{3}^{f} D_{6}-\varpi \frac{\partial}{\partial t} D_{5} & =D_{1}\left\{k^{f}\left(\xi \varpi k^{s} \frac{\partial}{\partial t}+k^{f} Q_{5}^{s}\right)-\left(\alpha^{f}+\beta^{f}\right)\left[\left(k^{s}\right)^{2} \Delta Q_{1}^{f}-P_{2} Q_{2}^{s}\right]\right\}, \\
\varepsilon_{i j k} P_{, k j} & =0
\end{aligned}
$$

we have that $u_{r}^{s}, v_{r}^{f}, \phi_{r}^{s}, \nu_{r}^{f}$ and $\pi^{f}$ are solutions of the basic system of equations (2.1), and the proof is complete.

\section{Fundamental solution for steady state vibrations}

In this section we use the representation described in the previous section in order to determine the fundamental solution of equations of motion for the case of steady vibrations. We suppose that

$$
\begin{aligned}
& F_{r}^{\sigma}=\operatorname{Re}\left[F_{r}^{* \sigma}(\mathbf{x}) \mathrm{e}^{-\mathrm{i} \omega \mathrm{t}}\right], \quad L_{r}^{\sigma}=\operatorname{Re}\left[L_{r}^{* \sigma}(\mathbf{x}) \mathrm{e}^{-\mathrm{i} \omega \mathrm{t}}\right], \\
& u_{r}^{\sigma}=\operatorname{Re}\left[u_{r}^{* \sigma}(\mathbf{x}) \mathrm{e}^{-\mathrm{i} \omega \mathrm{t}}\right], \quad \phi_{r}^{\sigma}=\operatorname{Re}\left[\phi_{r}^{* \sigma}(\mathbf{x}) \mathrm{e}^{-\mathrm{i} \omega \mathrm{t}}\right], \\
& \pi^{f}=\operatorname{Re}\left[\pi^{* f}(\mathbf{x}) \mathrm{e}^{-\mathrm{i} \omega \mathrm{t}}\right], \quad(\sigma=s, f),
\end{aligned}
$$

where $i$ is the imaginary unit and $\omega>0$ is the frequency of the vibration.

Regarding the propagation of plane harmonic waves in micropolar materials we have to say that the micropolar effects become important in highfrequency and short wave-length regions of waves (Eringen, 1999). In the theory of micropolar mixtures introduced by Eringen (2003) this aspect has been studied by Singh \& Tomar (2006). In the present paper we consider that the frequency is high enough for the solution for steady state vibrations to exist.

Let us introduce the differential operators: 


$$
\begin{aligned}
& Q_{1}^{* s}(\Delta)=\left(\mu^{s}+k^{s}\right) \Delta+\mathrm{i} \omega \xi-\rho^{s} \omega^{2}, \quad Q_{2}^{* s}(\Delta)=\gamma^{s} \Delta-2 k^{s}+\mathrm{i} \omega \varpi-\rho^{s} \omega^{2} j^{s}, \\
& Q_{3}^{* s}(\Delta)=\left(\lambda^{s}+\mu^{s}\right) \Delta+Q_{1}^{* s}, \quad Q_{4}^{* s}(\Delta)=\left(\alpha^{s}+\beta^{s}\right) \Delta+Q_{2}^{* s}, \\
& Q_{5}^{* s}(\Delta)=\left(k^{s}\right)^{2} \Delta+Q_{1}^{* s} Q_{2}^{* s}, \quad Q_{1}^{* f}(\Delta)=\left(\mu^{f}+k^{f}\right) \Delta-\xi+\mathrm{i} \rho^{f} \omega, \\
& Q_{2}^{* f}(\Delta)=\gamma^{f} \Delta-2 k^{f}-\varpi+\mathrm{i} \rho^{f} \omega j^{f}, Q_{3}^{* f}(\Delta)=\left(\alpha^{f}+\beta^{f}\right) \Delta+Q_{2}^{* f}, \\
& Q_{4}^{* f}(\Delta)=\left(k^{f}\right)^{2} \Delta+Q_{1}^{* f} Q_{2}^{* f}, P_{1}^{*}(\Delta)=-\mathrm{i} \omega \varpi^{2}-Q_{2}^{* f} Q_{2}^{* s}, \\
& P_{2}^{*}(\Delta)=-\mathrm{i} \omega \xi^{2}-Q_{1}^{* f} Q_{1}^{* s}, \quad D_{1}^{*}(\Delta)=\mathrm{i} \omega \varpi^{2}+Q_{3}^{* f} Q_{4}^{* s}, \\
& D_{2}^{*}(\Delta)=Q_{5}^{* s} Q_{4}^{* f}-2 \mathrm{i} \omega \xi \varpi k^{s} k^{f} \Delta+\mathrm{i} \omega\left(\varpi^{2} Q_{1}^{* s} Q_{1}^{* f}+\xi^{2} Q_{2}^{* s} Q_{2}^{* f}\right)+\xi^{2} \omega^{2} \varpi^{2}, \\
& D_{3}^{*}(\Delta)=D_{2}^{*}-\left(Q_{4}^{* f} Q_{2}^{* s}+\mathrm{i} \omega \varpi^{2} Q_{1}^{* f}\right) Q_{3}^{* s}, \\
& D_{4}^{*}(\Delta)=Q_{4}^{* f} Q_{3}^{* f}\left[\left(k^{s}\right)^{2}-\left(\alpha^{s}+\beta^{s}\right) Q_{1}^{* s}\right]-2 \mathrm{i} \omega \xi \varpi k^{s} k^{f} Q_{3}^{* f}- \\
& -\mathrm{i} \omega \varpi^{2} Q_{1}^{* s}\left[\left(k^{f}\right)^{2}-\left(\alpha^{f}+\beta^{f}\right) Q_{1}^{* f}\right]-\mathrm{i} \omega \xi^{2}\left(\alpha^{s}+\beta^{s}\right) Q_{2}^{* f} Q_{3}^{* f}+\left(\alpha^{f}+\beta^{f}\right) \xi^{2} \omega^{2} \varpi^{2}, \\
& D_{5}^{*}(\Delta)=Q_{5}^{*} \varpi\left(k^{f}\right)^{2}+\xi k^{s} k^{f}\left(-\mathrm{i} \omega \varpi^{2}+Q_{3}^{* f} Q_{4}^{* s}\right)+\varpi\left\{\left[\left(\alpha^{s}+\beta^{s}\right) Q_{2}^{* f}+\right.\right. \\
& \left.\left.\quad+\left(\alpha^{f}+\beta^{f}\right) Q_{2}^{* s}+\left(\alpha^{f}+\beta^{f}\right)\left(\alpha^{s}+\beta^{s}\right) \Delta\right] P_{2}^{*}+\left(k^{s}\right)^{2} Q_{1}^{* f} Q_{2}^{* f}\right\}, \\
& D_{6}^{*}(\Delta)=Q_{5}^{* s} Q_{4}^{* s}\left[\left(k^{f}\right)^{2}-\left(\alpha^{f}+\beta^{f}\right) Q_{1}^{* f}\right]-2 \mathrm{i} \omega \xi \varpi k^{s} k^{f} Q_{4}^{* s}- \\
& -\mathrm{i} \omega \varpi^{2} Q_{1}^{* f}\left[\left(k^{s}\right)^{2}-\left(\alpha^{s}+\beta^{s}\right) Q_{1}^{* s}\right]-\mathrm{i} \omega \xi^{2}\left(\alpha^{f}+\beta^{f}\right) Q_{2}^{* s} Q_{4}^{* s}+\omega^{2} \xi^{2} \varpi^{2}\left(\alpha^{s}+\beta^{s}\right) .
\end{aligned}
$$

We introduce the differential matrix operator

$$
\mathbf{D}\left(\frac{\partial}{\partial \mathbf{x}}\right)=\left\|D_{m n}\left(\frac{\partial}{\partial \mathbf{x}}\right)\right\|_{13 \times 13},
$$

where

$$
\begin{aligned}
& D_{r j}=Q_{1}^{* s} \delta_{r j}+\left(\lambda^{s}+\mu^{s}\right) \frac{\partial^{2}}{\partial x_{r} \partial x_{j}}, \\
& D_{r ; 3+j}=-D_{3+j ; r}=k^{s} \varepsilon_{r k j} \frac{\partial}{\partial x_{k}}, \\
& D_{r ; j+6}=D_{j+6 ; r}=-\mathrm{i} \omega \xi, \\
& D_{r ; j+9}=D_{j+9 ; r}=D_{r ; 13}=D_{13 ; r}=D_{r+3 ; j+6}=D_{j+6 ; r+3}=0, \\
& D_{r+3 ; 13}=D_{13 ; r+3}=D_{r+9 ; 13}=D_{13 ; r+9}=D_{13 ; 13}=0, \\
& D_{r+3 ; j+3}=Q_{2}^{* s} \delta_{r j}+\left(\alpha^{s}+\beta^{s}\right) \frac{\partial^{2}}{\partial x_{r} \partial x_{j}}, \\
& D_{r+3 ; j+9}=D_{j+9 ; r+3}=-\mathrm{i} \omega \varpi, \\
& D_{r+6 ; j+6}=-\mathrm{i} \omega Q_{1}^{* f} \delta_{r j}, \\
& D_{r+6 ; j+9}=-D_{j+9 ; r+6}=-\mathrm{i} \omega k^{f} \varepsilon_{r k j} \frac{\partial}{\partial x_{k}}, \\
& D_{r+9 ; j+9}=-\mathrm{i} \omega Q_{2}^{* f} \delta_{r j}-\mathrm{i} \omega\left(\alpha^{f}+\beta^{f}\right) \frac{\partial^{2}}{\partial x_{r} \partial x_{j}},
\end{aligned}
$$




$$
\begin{aligned}
& D_{r+6 ; 13}=-\frac{1}{\chi} D_{13 ; r+6}=-\frac{\partial}{\partial x_{r}} \\
& \chi=\left(\lambda^{s}+2 \mu^{s}+k^{s}\right)^{-1} \prod_{\sigma=s, f}\left[\left(\alpha^{\sigma}+\beta^{\sigma}+\gamma^{\sigma}\right) \gamma^{\sigma}\left(k^{\sigma}+\mu^{\sigma}\right)\right]^{-1} .
\end{aligned}
$$

If we introduce the thirteen-dimensional vectors $\mathbf{U}=\left(\mathbf{u}^{* s}, \boldsymbol{\phi}^{* s}, \mathbf{u}^{* f}, \boldsymbol{\phi}^{* f}, \pi^{* f}\right)$ and $\mathbf{F}=\left(\mathbf{F}^{* s}, \mathbf{L}^{* s}, \mathbf{F}^{* f}, \mathbf{L}^{* f}, 0\right)$, it is easy to see that the basic system of equations which describes the behaviour of the amplitudes $\mathbf{U}$ of the steady vibrations can be written in the form

$$
\mathbf{D}\left(\frac{\partial}{\partial \mathbf{x}}\right) \mathbf{U}=-\mathbf{F} .
$$

Definition 4.1. Let be $\mathbf{y} \in \mathbf{E}^{3}$. A fundamental solution of the system (4.4) is a matrix $\mathbf{\Gamma}(\mathbf{x}, \mathbf{y} ; \omega)=\left\|\Gamma_{r j}\right\|_{13 \times 13}$ which satisfies the condition (Hörmander, 1964)

$$
\mathbf{D}\left(\frac{\partial}{\partial \mathbf{x}}\right) \boldsymbol{\Gamma}(\mathbf{x}, \mathbf{y} ; \omega)=-\delta(\mathbf{x}-\mathbf{y}) \mathbf{I}, \quad \mathbf{x} \in \mathbf{E}^{3},
$$

where $\delta(\cdot)$ is the Dirac delta and $\mathbf{I}=\left\|\delta_{r j}\right\|_{13 \times 13}$ is the unit matrix.

According to the general theory of the fundamental solutions of the differential operators (Kythe, 1996), we have to say that a fundamental solution is unique up to a matrix which has as columns solutions of the homogeneous system

$$
\mathbf{D}\left(\frac{\partial}{\partial \mathbf{x}}\right) \mathbf{U}=0
$$

As a consequence of the Theorem 3.1 we obtain the following result:

Theorem 4.1. Let

$$
\begin{aligned}
u_{r}^{* s}= & \Delta Q_{3}^{* s} D_{1}^{*}\left(\mathrm{i} \omega \varpi^{2} Q_{1}^{* f}+Q_{4}^{* f} Q_{2}^{* s}\right) G_{r}^{* s}+D_{1}^{*} D_{3}^{*} G_{j, r j}^{* s}+ \\
& +\varepsilon_{r j k} \Delta Q_{3}^{* s} D_{1}^{*}\left(k^{s} Q_{4}^{* f}-\mathrm{i} \omega \xi \varpi k^{f}\right) H_{j, k}^{* s}+ \\
& +\Delta Q_{3}^{* s} D_{1}^{*}\left(\varpi k^{s} k^{f} \Delta+\xi P_{1}^{*}\right) G_{r}^{* f}-Q_{3}^{* s} D_{1}^{*}\left(\varpi k^{s} k^{f} \Delta+\xi P_{1}^{*}\right) G_{j, r j}^{* f}- \\
& -\varepsilon_{r j k} \Delta Q_{3}^{* s} D_{1}^{*}\left(\varpi k^{s} Q_{1}^{* f}+\xi k^{f} Q_{2}^{* s}\right) H_{j, k}^{* f}-\xi P_{, r}^{*}, \\
\phi_{r}^{* s}= & \varepsilon_{r j k} \Delta Q_{3}^{* s} D_{1}^{*}\left(k^{s} Q_{4}^{* f}-\mathrm{i} \omega \xi \varpi k^{f}\right) G_{j, k}^{* s}+ \\
& +\Delta Q_{3}^{* s} D_{1}^{*}\left[\left(k^{f}\right)^{2} \Delta Q_{1}^{* s}-P_{2}^{*} Q_{2}^{* f}\right] H_{r}^{* s}+\Delta Q_{3}^{* s} D_{4}^{*} H_{j, r j}^{* s}- \\
& -\varepsilon_{r j k} D_{1}^{*} \Delta Q_{3}^{* s}\left(\varpi k^{f} Q_{1}^{* s}+\xi k^{s} Q_{2}^{* f}\right) G_{j, k}^{* f}+ \\
& +\Delta Q_{3}^{* s} D_{1}^{*}\left(\xi k^{s} k^{f} \Delta+\varpi P_{2}^{*}\right) H_{r}^{* f}-\Delta Q_{3}^{* s} D_{5}^{*} H_{j, r j}^{* f},
\end{aligned}
$$




$$
\begin{aligned}
u_{r}^{* f}= & \Delta Q_{3}^{* s} D_{1}^{*}\left(\varpi k^{s} k^{f} \Delta+\xi P_{1}^{*}\right) G_{r}^{* s}-Q_{3}^{* s} D_{1}^{*}\left(\varpi k^{s} k^{f} \Delta+\xi P_{1}^{*}\right) G_{j, r j}^{* s}- \\
& -\varepsilon_{r j k} \Delta Q_{3}^{* s} D_{1}^{*}\left(\varpi k^{f} Q_{1}^{* s}+\xi k^{s} Q_{2}^{* f}\right) H_{j, k}^{* s}+ \\
& +\frac{\mathrm{i}}{\omega} \Delta Q_{3}^{* s} D_{1}^{*}\left(\mathrm{i} \omega \varpi^{2} Q_{1}^{* s}+Q_{5}^{* s} Q_{2}^{* f}\right) G_{r}^{* f}-\frac{\mathrm{i}}{\omega} Q_{3}^{* s} D_{1}^{*}\left(\mathrm{i} \omega \varpi^{2} Q_{1}^{* s}+Q_{5}^{* s} Q_{2}^{* f}\right) G_{j, r j}^{* f}+ \\
& +\frac{\mathrm{i}}{\omega} \varepsilon_{r j k} \Delta Q_{3}^{* s} D_{1}\left(-\mathrm{i} \omega \xi \varpi k^{s}+k^{f} Q_{5}^{* s}\right) H_{j, k}^{* f}+\frac{\mathrm{i}}{\omega} Q_{3}^{* s} P_{, r}^{*}, \\
\phi_{r}^{* f}= & -\varepsilon_{r j k} \Delta Q_{3}^{* s} D_{1}^{*}\left(\varpi k^{s} Q_{1}^{* f}+\xi k^{f} Q_{2}^{* s}\right) G_{j, k}^{* s}+ \\
& +\Delta Q_{3}^{* s} D_{1}^{*}\left(\xi k^{s} k^{f} \Delta+\varpi P_{2}^{*}\right) H_{r}^{* s}-\Delta Q_{3}^{* s} D_{5}^{*} H_{j, r j}^{* s}+ \\
& +\frac{\mathrm{i}}{\omega} \varepsilon_{r j k} \Delta Q_{3}^{* s} D_{1}^{*}\left(-\mathrm{i} \omega \xi \varpi k^{s}+k^{f} Q_{5}^{* s}\right) G_{j, k}^{* f}+ \\
& +\frac{\mathrm{i}}{\omega} \Delta Q_{3}^{* s} D_{1}\left[\left(k^{s}\right)^{2} \Delta Q_{1}^{* f}-P_{2}^{*} Q_{2}^{* s}\right] H_{r}^{* f}+\frac{\mathrm{i}}{\omega} \Delta Q_{3}^{* s} D_{6}^{*} H_{j, r j}^{* f}, \\
\pi^{* f}= & -\mathrm{i} \omega \xi D_{1}^{*} D_{2}^{*} G_{r, r}^{* s}-Q_{3}^{* s} D_{1}^{*} D_{2}^{*} G_{r, r}^{* f}+\left(\mathrm{i} \xi^{2} \omega+Q_{1}^{* f} Q_{3}^{* f}\right) P^{*},
\end{aligned}
$$

where $G_{r}^{* s}, G_{r}^{* f}, H_{r}^{* s}, F_{r}^{* f}$ and $P^{*}$ satisfy

$\Delta Q_{3}^{* s} D_{1}^{*} D_{2}^{*} G_{r}^{* \sigma}=-F_{r}^{* \sigma}, \Delta Q_{3}^{* s} D_{1}^{*} D_{2}^{*} H_{r}^{* \sigma}=-L_{r}^{* \sigma}, \Delta Q_{3}^{* s} P^{*}=0(\sigma=s, f)$.

Then $u_{r}^{* s}, u_{r}^{* f}, \phi_{r}^{* s}, \phi_{r}^{* f}$ and $\pi^{* f}$ satisfy the equations (4.4).

We denote by $k_{n}^{2}, n=1,2$ and respectively, by $k_{m}^{2}, m=3,4,5,6$ the roots of the equations

$$
D_{1}^{*}(-k)=0, D_{2}^{*}(-k)=0
$$

It is convenient to write

$$
Q_{3}^{* s}(\Delta)=\left(\lambda^{s}+2 \mu^{s}+k^{s}\right)\left(\Delta+k_{7}^{2}\right)
$$

where $k_{7}$ is the complex number defined by

$$
k_{7}^{2}=-\frac{1}{\lambda^{s}+2 \mu^{s}+k^{s}}\left(\rho^{s} \omega^{2}-\mathrm{i} \omega \xi\right) .
$$

We assume that $k_{n}^{2} \neq k_{m}^{2}$, for $n \neq m, n, m=1,2, \ldots, 7$, and we choose the complex number $k_{n}$ such that $\operatorname{Im}\left[k_{n}\right] \geq 0$, for $n=1,2, \ldots, 7$.

With the help of these quantities, we can rewrite the equations (4.8) in the following form

$$
\begin{gathered}
\Delta \prod_{n=1}^{7}\left(\Delta+k_{n}^{2}\right) G_{r}^{* \sigma}=-\chi F_{r}^{* \sigma}, \quad \Delta \prod_{n=1}^{7}\left(\Delta+k_{n}^{2}\right) H_{r}^{* \sigma}=-\chi L_{r}^{* \sigma}, \\
\Delta\left(\Delta+k_{7}^{2}\right) P^{*}=0 \quad(\sigma=s, f) .
\end{gathered}
$$


Proposition 4.1. Assume that $F_{r}^{* s}=\delta_{r k} \delta(\mathbf{x}-\mathbf{y}), L_{r}^{* s}=0, F_{r}^{* f}=0$ and $L_{r}^{* f}=0$. Then, the equations (4.12) have the solution $G_{r}^{* s}=\delta_{r k} E(\mathbf{x}, \mathbf{y} ; \omega)$, $H_{r}^{* s}=0, G_{r}^{* f}=0, H_{r}^{* f}=0, P^{*}=0$, where

$$
\begin{aligned}
& E(\mathbf{x}, \mathbf{y} ; \omega)=\sum_{n=1}^{7} c_{n} E_{n}(\mathbf{x}, \mathbf{y} ; \omega), \quad E_{n}=\frac{\chi}{4 \pi \varrho k_{n}^{2}}\left(1-e^{\mathrm{i} k_{n} \varrho}\right), \\
& c_{n}^{-1}=\prod_{m=1, m \neq n}^{7}\left(k_{n}^{2}-k_{m}^{2}\right) \quad n=1,2, \ldots, 7, \quad \varrho^{2}=\left(x_{r}-y_{r}\right)\left(x_{r}-y_{r}\right) .
\end{aligned}
$$

Proof. First of all, we remark that

$$
\Delta\left(\Delta+k_{n}^{2}\right) E_{n}=-\chi \delta(\mathbf{x}-\mathbf{y}) .
$$

Taking into account the relations

$$
\begin{gathered}
\sum_{n=1}^{7} c_{n}=0, \sum_{n=m}^{7} c_{n} \prod_{l=1}^{m-1}\left(k_{l}^{2}-k_{n}^{2}\right)=0 \text { for } m=2,3, \ldots, 6 \\
\left(\Delta+k_{n}^{2}\right) E_{m}=\chi \delta(\mathbf{x}-\mathbf{y})+\left(k_{n}^{2}-k_{m}^{2}\right) E_{m} \text { for } n, m=1,2, \ldots, 7,
\end{gathered}
$$

and the method presented in the paper (Svanadze, 1996), we have

$$
\Delta \prod_{n=1}^{7}\left(\Delta+k_{n}^{2}\right) E(\mathbf{x}, \mathbf{y} ; \omega)=-\chi \delta(\mathbf{x}-\mathbf{y})
$$

and the proof is complete.

We denote by $\left(u_{r}^{* s(k)}, \phi_{r}^{* s(k)}, u_{r}^{* f(k)}, \phi_{r}^{* f(k)}\right)$ the amplitudes of displacements caused by the concentrated loads $F_{r}^{* s}=\delta_{r k} \delta(\mathbf{x}-\mathbf{y}), L_{r}^{* s}=0, F_{r}^{* f}=0$ and $L_{r}^{* f}=0$. In view of the relations (4.7) we get

$$
\begin{aligned}
& u_{r}^{* s(k)}=\Delta Q_{3}^{* s} D_{1}^{*}\left(\mathrm{i} \omega \varpi^{2} Q_{1}^{* f}+Q_{4}^{* f} Q_{2}^{* s}\right) \delta_{r k} E+D_{1}^{*} D_{3}^{*} E_{, r k}, \\
& \phi_{r}^{*(k)}=\varepsilon_{r k l} \Delta Q_{3}^{* s} D_{1}^{*}\left(k^{s} Q_{4}^{* f}-\mathrm{i} \omega \xi \varpi k^{f}\right) E_{, l}, \\
& u_{r}^{* f(k)}=\Delta Q_{3}^{* s} D_{1}^{*}\left(\varpi k^{s} k^{f} \Delta+\xi P_{1}^{*}\right) \delta_{r k} E-Q_{3}^{* s} D_{1}^{*}\left(\varpi k^{s} k^{f} \Delta+\xi P_{1}^{*}\right) E_{, r k}, \\
& \phi_{r}^{* f(k)}=-\varepsilon_{r k l} \Delta Q_{3}^{* s} D_{1}^{*}\left(\varpi k^{s} Q_{1}^{* f}+\xi k^{f} Q_{2}^{* s}\right) E_{, l}, \\
& \pi^{* f(k)}=-\mathrm{i} \omega \xi D_{1}^{*} D_{2}^{*} E_{, k} .
\end{aligned}
$$

Corresponding to the concentrated loads, $F_{r}^{* s}=0, L_{r}^{* s}=\delta_{r k} \delta(\mathbf{x}-\mathbf{y})$, $F_{r}^{* f}=0$ and $L_{r}^{* f}=0$, we have the following amplitude of displacement, 
denoted by $\left(u_{r}^{* s(3+k)}, \phi_{r}^{* s(3+k)}, u_{r}^{* f(3+k)}, \phi_{r}^{* f(3+k)}\right)$,

$u_{r}^{* s(3+k)}=\varepsilon_{r k l} \Delta Q_{3}^{* s} D_{1}^{*}\left(k^{s} Q_{4}^{* f}-i \omega \xi \varpi k^{f}\right) E_{, l}$,

$\phi_{r}^{* s(3+k)}=\Delta Q_{3}^{* s} D_{1}^{*}\left[\left(k^{f}\right)^{2} \Delta Q_{1}^{* s}-P_{2}^{*} Q_{2}^{* f}\right] \delta_{r k} E+\Delta Q_{3}^{* s} D_{4}^{*} E_{, r k}$,

$u_{r}^{* f(3+k)}=-\varepsilon_{r k l} \Delta Q_{3}^{* s} D_{1}^{*}\left(\varpi k^{f} Q_{1}^{* s}+\xi k^{s} Q_{2}^{* f}\right) E_{, l}$,

$\phi_{r}^{* f(3+k)}=\Delta Q_{3}^{* s} D_{1}^{*}\left(\xi k^{s} k^{f} \Delta+\varpi P_{2}^{*}\right) \delta_{r k} E-\Delta Q_{3}^{* s} D_{5}^{*} E_{, r k}$,

$\pi^{* f(3+k)}=0$.

If $F_{r}^{* s}=0, L_{r}^{* s}=0, F_{r}^{* f}=\delta_{r k} \delta(\mathbf{x}-\mathbf{y})$ and $L_{r}^{* f}=0$, then the corresponding displacement vectors denoted by $\left(u_{r}^{* s(6+k)}, \phi_{r}^{* s(6+k)}, u_{r}^{* f(6+k)}, \phi_{r}^{* f(6+k)}\right)$ are

$$
\begin{aligned}
u_{r}^{* s(6+k)}= & \Delta Q_{3}^{* s} D_{1}^{*}\left(\varpi k^{s} k^{f} \Delta+\xi P_{1}^{*}\right) \delta_{r k} E-Q_{3}^{* s} D_{1}^{*}\left(\varpi k^{s} k^{f} \Delta+\xi P_{1}^{*}\right) E_{, r k}, \\
\phi_{r}^{* s(6+k)}= & -\varepsilon_{r k l} D_{1}^{*} \Delta Q_{3}^{* s}\left(\varpi k^{f} Q_{1}^{* s}+\xi k^{s} Q_{2}^{* f}\right) E_{, l} \\
u_{r}^{* f(6+k)}= & \frac{\mathrm{i}}{\omega} \Delta Q_{3}^{* s} D_{1}^{*}\left(\mathrm{i} \omega \varpi^{2} Q_{1}^{* s}+Q_{5}^{* s} Q_{2}^{* f}\right) \delta_{r k} E- \\
& \quad-\frac{\mathrm{i}}{\omega} Q_{3}^{* s} D_{1}^{*}\left(\mathrm{i} \omega \varpi^{2} Q_{1}^{* s}+Q_{5}^{* s} Q_{2}^{* f}\right) E_{, r k}, \\
\phi_{r}^{* f(6+k)}= & \frac{\mathrm{i}}{\omega} \varepsilon_{r k l} \Delta Q_{3}^{* s} D_{1}^{*}\left(-\mathrm{i} \omega \xi \varpi k^{s}+k^{f} Q_{5}^{* s}\right) E_{, l}, \\
\pi^{* f(6+k)=}= & -Q_{3}^{* s} D_{1}^{*} D_{2}^{*} E_{, k} .
\end{aligned}
$$

Finally, if $F_{r}^{* s}=0, L_{r}^{* s}=0, F_{r}^{* f}=0$ and $L_{r}^{* f}=\delta_{r k} \delta(\mathbf{x}-\mathbf{y})$, then we have for the displacement vectors $\left(u_{r}^{* s(9+k)}, \phi_{r}^{* s(9+k)}, u_{r}^{* f(9+k)}, \phi_{r}^{* f(9+k)}\right)$ the expressions

$$
\begin{aligned}
u_{r}^{* s(9+k)} & =-\varepsilon_{r k l} \Delta Q_{3}^{* s} D_{1}^{*}\left(\varpi k^{s} Q_{1}^{* f}+\xi k^{f} Q_{2}^{* s}\right) E_{, l}, \\
\phi_{r}^{* s(9+k)} & =\Delta Q_{3}^{* s} D_{1}^{*}\left(\xi k^{s} k^{f} \Delta+\varpi P_{2}^{*}\right) \delta_{r k} E-\Delta Q_{3}^{* s} D_{5}^{*} E_{, r k}, \\
u_{r}^{* f(9+k)} & =\frac{\mathrm{i}}{\omega} \varepsilon_{r k l} \Delta Q_{3}^{* s} D_{1}\left(-\mathrm{i} \omega \xi \varpi k^{s}+k^{f} Q_{5}^{* s}\right) E_{, l}, \\
\phi_{r}^{* f(9+k)} & =\frac{\mathrm{i}}{\omega} \Delta Q_{3}^{* s} D_{1}\left[\left(k^{s}\right)^{2} \Delta Q_{1}^{* f}-P_{2}^{*} Q_{2}^{* s}\right] \delta_{r k} E+\frac{\mathrm{i}}{\omega} \Delta Q_{3}^{* s} D_{6}^{*} E_{, r k}, \\
\pi^{* f(9+k)} & =0 .
\end{aligned}
$$

From the above discussion we can conclude: 
Theorem 4.1. The matrix $\mathbf{\Gamma}(\mathbf{x}, \mathbf{y} ; \omega)$ defined by

$$
\begin{aligned}
& \Gamma_{r ; k}=u_{r}^{* s(k)}, \Gamma_{3+r ; k}=\phi_{r}^{* s(k)}, \Gamma_{6+r ; k}=u_{r}^{* f(k)}, \Gamma_{9+r ; k}=\phi_{r}^{* f(k)}, \\
& \Gamma_{r ; 3+k}=u_{r}^{* s(3+k)}, \Gamma_{3+r ; 3+k}=\phi_{r}^{* s(3+k)}, \Gamma_{6+r ; 3+k}=u_{r}^{* f(3+k)}, \\
& \Gamma_{9+r ; 3+k}=\phi_{r}^{* f(3+k)}, \Gamma_{r ; 6+k}(\mathbf{x}, \omega)=u_{r}^{* s(6+k)}, \Gamma_{3+r ; 6+k}=\phi_{r}^{* s(6+k)}, \\
& \Gamma_{6+r ; 6+k}=u_{r}^{* f(6+k)}, \Gamma_{9+r ; 6+k}=\phi_{r}^{* f(6+k)}, \Gamma_{r ; 9+k}(\mathbf{x}, \omega)=u_{r}^{* s(9+k)}, \\
& \Gamma_{3+r ; 9+k}=\phi_{r}^{* s(9+k)}, \Gamma_{6+r ; 9+k}=u_{r}^{* f(9+k)}, \Gamma_{9+r ; 9+k}=\phi_{r}^{* f(9+k)}, \\
& -\Gamma_{k ; 13}=\Gamma_{13 ; k}=\pi^{* f(k)},-\Gamma_{6+k ; 13}=\Gamma_{13 ; 6+k}=\pi^{* f(6+k)}, \\
& \Gamma_{3+k ; 13}=\Gamma_{13 ; 3+k}=\Gamma_{9+k ; 13}=\Gamma_{13 ; 9+k}=\Gamma_{13 ; 13}=0
\end{aligned}
$$

is a fundamental solution of the system (4.4).

\section{Basic properties of the matrix $\Gamma(\mathrm{x}, \mathrm{y} ; \omega)$}

In this section we point out some basic properties of the fundamental solution constructed in the previous section. These basic properties of fundamental matrix are useful if we want to apply the potential method for the framework theory.

Let us first note that

Proposition 5.1. The fundamental matrix $\boldsymbol{\Gamma}(\mathbf{x}, \mathbf{y} ; \omega)$ is so that

(i) $\boldsymbol{\Gamma}(\mathbf{x}, \mathbf{y} ; \omega)=\boldsymbol{\Gamma}^{T}(\mathbf{y}, \mathbf{x} ; \omega)$;

(ii) If $\mathbf{x} \neq \mathbf{y}$, then each column $\boldsymbol{\Gamma}^{(m)}(\mathbf{x}, \mathbf{y} ; \omega),(m=1,2, \ldots, 13)$ of the matrix $\Gamma$ satisfies at $\mathbf{x}$ the homogeneous system

$$
\mathbf{D}\left(\frac{\partial}{\partial \mathbf{x}}\right) \boldsymbol{\Gamma}^{(m)}(\mathbf{x}, \mathbf{y} ; \omega)=0 .
$$

Lemma 5.1. The function $E$ has the following properties:

(i) $\frac{\partial E}{\partial x_{r}^{s_{1}} \partial x_{l}^{s_{2}} \partial x_{k}^{s_{3}}}=O(\varrho)(\varrho \rightarrow 0)$, for all even $s \leq 11$;

(ii) $\frac{\partial E}{\partial x_{r}^{s_{1}} \partial x_{l}^{s_{2}} \partial x_{k}^{s_{3}}}=\mathrm{const}+O\left(\varrho^{2}\right)(\varrho \rightarrow 0)$, for all odd $s \leq 11$;

(iii) $\frac{\partial E}{\partial x_{r}^{s_{1}} \partial x_{l}^{s_{2}} \partial x_{k}^{s_{3}}}=O\left(\varrho^{13-s}\right)(\varrho \rightarrow 0)$, for all $s \geq 12$;

where $s_{1}, s_{2}, s_{3} \in \mathbb{N}^{*}$ and $s=s_{1}+s_{2}+s_{3}$. 
Proof. It is easy to see that in the neighbourhood of $\mathbf{y}$, we have

$$
\frac{1-e^{\mathrm{i} k_{n} \varrho}}{k_{n}^{2} \varrho}=-\frac{\mathrm{i}}{k_{n}}+\sum_{m=0}^{\infty} \frac{\left(\mathrm{i} k_{n}\right)^{m}}{(m+2) !} \varrho^{m+1} .
$$

On the other hand, we can deduce that

$$
\sum_{n=1}^{7} c_{n} k_{n}^{2 p}=0, \text { for } p=0,1, \ldots, 5 \text { and } \sum_{n=1}^{7} c_{n} k_{n}^{12}=1 .
$$

Thus, we obtain

$E=\frac{\chi}{4 \pi}\left[-\mathrm{i} \sum_{n=1}^{7} \frac{c_{n}}{k_{n}}+\sum_{n=1}^{7} \sum_{m=1}^{6} \frac{c_{n}\left(\mathrm{i} k_{n}\right)^{2 m-1}}{(2 m+1) !} \varrho^{2 m}+\frac{1}{14 !} \varrho^{13}+\sum_{n=1}^{7} \sum_{m=13}^{\infty} \frac{c_{n}\left(\mathrm{i} k_{n}\right)^{m}}{(m+2) !} \varrho^{m+1}\right]$.

Using this relation we obtain the conclusions of lemma.

Let us introduce the matrix $\boldsymbol{\Pi}$ defined by

$$
\begin{aligned}
& \Pi_{r ; k}=\frac{1}{4 \pi}\left(\frac{1}{\mu^{s}+k^{s}}-\frac{1}{2 a^{s}}\right) \delta_{r k} \frac{1}{\varrho}+\frac{1}{8 \pi a^{s}} \frac{x_{r} x_{k}}{\varrho^{3}}, \\
& \Pi_{3+r ; k}=\Pi_{6+r ; k}=\Pi_{9+r ; k}=\Pi_{13 ; k}=0, \\
& \Pi_{3+r ; 3+k}=\frac{1}{4 \pi}\left(\frac{1}{\gamma^{s}}-\frac{1}{2 b^{s}}\right) \delta_{r k} \frac{1}{\varrho}+\frac{1}{8 \pi b^{s}} \frac{x_{r} x_{k}}{\varrho^{3}}, \\
& \Pi_{r ; 3+k}=\Pi_{6+r ; 3+k}=\Pi_{9+r ; 3+k}=\Pi_{13 ; 3+k}=0, \\
& \Pi_{6+r ; 6+k}=\frac{\mathrm{i}}{8 \pi \omega\left(\mu^{f}+k^{f}\right)}\left(\delta_{r k} \frac{1}{\varrho}+\frac{x_{r} x_{k}}{\varrho^{3}}\right), \\
& \Pi_{r ; 6+k}=\Pi_{3+r ; 6+k}=\Pi_{9+r ; 6+k}=0, \Pi_{13 ; 6+k}=-\Pi_{6+k ; 13}=-\frac{1}{4 \pi} \frac{x_{k}}{\varrho^{3}}, \\
& \Pi_{9+r ; 9+k}=\frac{\mathrm{i}}{4 \pi \omega}\left(\frac{1}{\gamma^{s}}-\frac{1}{2 b^{s}}\right) \delta_{r k} \frac{1}{\varrho}+\frac{\mathrm{i}}{8 \pi \omega b^{s}} \frac{x_{r} x_{k}}{\varrho^{3}}, \\
& \Pi_{r ; 9+k}=\Pi_{3+r ; 9+k}=\Pi_{6+r ; 9+k}=\Pi_{13 ; 3+k}=0, \\
& \Pi_{k ; 13}=\Pi_{3+k ; 13}=\Pi_{9+k ; 13}=\Pi_{13 ; 13}=0,
\end{aligned}
$$

where

$$
\begin{gathered}
a^{s}=\frac{\left(\mu^{s}+k^{s}\right)\left(\lambda^{s}+2 \mu^{s}+k^{s}\right)}{\lambda^{s}+\mu^{s}} \\
b^{\sigma}=\frac{\gamma^{\sigma}\left(\alpha^{\sigma}+\beta^{\sigma}+\gamma^{\sigma}\right)}{\alpha^{\sigma}+\beta^{\sigma}}
\end{gathered}
$$

We can observe that

$$
\mathbf{\Pi}(\mathbf{x}, \mathbf{y} ; \omega)=\mathbf{\Pi}(\mathbf{y}, \mathbf{x} ; \omega), \quad \mathbf{\Pi}(\mathbf{x}, \mathbf{y} ; \omega)=\boldsymbol{\Pi}^{T}(\mathbf{x}, \mathbf{y} ; \omega) .
$$


We denote by $\boldsymbol{\Pi}^{(m)}(\mathbf{x}, \mathbf{y} ; \omega), \quad(m=1,2, \ldots, 13)$ the columns of the matrix $\Pi(\mathbf{x}, \mathbf{y} ; \omega)$.

In view of Lemma 5.1 we have

Proposition 5.2. The differences

$$
\boldsymbol{G}^{(m)}(\mathbf{x}, \mathbf{y} ; \omega)=\boldsymbol{\Gamma}^{(m)}(\mathbf{x}, \mathbf{y} ; \omega)-\boldsymbol{\Pi}^{(m)}(\mathbf{x}, \mathbf{y} ; \omega),
$$

remain bounded when $\mathbf{x}=\mathbf{y}$ and the first derivations of these differences have only a pole of the first order for $\mathbf{x}=\mathbf{y}$.

A thirteen-dimensional vector $\mathbf{U}=\left(\mathbf{u}^{* s}, \boldsymbol{\phi}^{* s}, \mathbf{u}^{* f}, \boldsymbol{\phi}^{* f}, \pi^{* f}\right)$ defined on $\mathbb{R}^{3}$, is called regular if $\mathbf{u}^{* s}, \boldsymbol{\phi}^{* s}, \mathbf{u}^{* f}, \boldsymbol{\phi}^{* f} \in \mathbf{C}^{2}\left(\mathbb{R}^{3}\right), \pi^{f} \in C^{1}\left(\mathbb{R}^{3}\right)$ and $u_{j, r}^{* s}, \phi_{j, r}^{* s}, u_{j, r}^{* f}$, $\phi_{j, r}^{* f}, \pi^{f} \in L^{2}(\Sigma(\mathbf{0}, R))$ for every $R>0$, and satisfies the asymptotic relations of the type

$$
u_{r}^{* \sigma}=O\left(\varrho^{-1}\right), \quad u_{r, l}^{* \sigma}=o\left(\varrho^{-1}\right), \quad \phi_{r}^{* \sigma}=o\left(\varrho^{-1}\right), \quad \phi_{r, l}^{* \sigma}=O\left(\varrho^{-1}\right),
$$

where $\Sigma(\mathbf{0}, R)$ is the sphere with its center at $\mathbf{0}$ and radius $R$.

As in (Gales, $2004 \mathrm{~b}$ ), we can prove that the system of equations (4.4) has a unique solution in the class of regular vectors.

Let remark that in view of the above proprieties and because we choose the complex numbers $k_{n}, n=1,2, \ldots, 7$ such that $\operatorname{Im}\left[k_{n}\right] \geq 0$, we can conclude that all the columns of the matrix $\mathbf{\Gamma}(\mathbf{x}, \mathbf{y} ; \omega)$ are regular vectors. Thus, the matrix $\boldsymbol{\Gamma}(\mathbf{x}, \mathbf{y} ; \omega)$ is the unique fundamental solution, up to a rearrangement of the columns, for which the columns are regular vectors.

\section{Solution for point load problem in cylindrical coordinates}

We consider an infinite micropolar solid-fluid mixture and a point $\mathbf{y}$ in the mixture. A concentrated force $\mathbf{F}^{* s}(\mathbf{x})=\delta(\mathbf{x}-\mathbf{y}) \mathbf{m}$ is applied to the mixture, where $\mathbf{m}$ is an unit vector. Based on the general solution described in Section 4, we give the solution of the problem corresponding to this point force.

We choose a system of the Cartesian axes such that the origin $O$ is in the point $\mathbf{y}$ and the direction of $O x_{3}$ is given by the unit vector $\mathbf{m}$. In the Cartesian coordinates $\left(x_{1}, x_{2}, x_{3}\right)$ we have $\mathbf{F}^{* s}(\mathbf{x})=\delta(\mathbf{x}) \mathbf{e}_{3}$.

Using (6.10) we find that the displacement of the solid is given by

$$
\mathbf{u}^{*}=\Delta Q_{3}^{* s} D_{1}^{*}\left(\mathrm{i} \omega \varpi^{2} Q_{1}^{* f}+Q_{4}^{* f} Q_{2}^{* s}\right) E \mathbf{e}_{3}+D_{1}^{*} D_{3}^{*} \operatorname{grad} E_{, 3},
$$

where $\operatorname{grad}=\frac{\partial}{\partial x_{k}} \mathbf{e}_{k}$ is the gradient operator and $\mathbf{e}_{k}$ are the unit vectors of the Cartesian axes. 
In the cylindrical coordinates $(r, \theta, z)$, because $E$ is independent by $\theta$, the components of the displacement of the solids are

$$
\begin{aligned}
& u_{r}^{* s}=D_{1}^{*} D_{3}^{*} \frac{z r}{\varrho^{2}}\left(\frac{\partial^{2} E}{\partial \varrho^{2}}-\frac{1}{\varrho} \frac{\partial E}{\partial \varrho}\right), \\
& u_{\theta}^{* s}=0, \\
& u_{z}^{* s}=\Delta Q_{3}^{* s} D_{1}^{*}\left(\mathrm{i} \omega \varpi^{2} Q_{1}^{* f}+Q_{4}^{* f} Q_{2}^{* s}\right) E+D_{1}^{*} D_{3}^{*} \frac{1}{\varrho^{2}}\left(\frac{\partial^{2} E}{\partial \varrho^{2}} z^{2}+\frac{r^{2}}{\varrho} \frac{\partial E}{\partial \varrho}\right),
\end{aligned}
$$

where we use that $\varrho^{2}=r^{2}+z^{2}$.

Similarly, we find

$$
\begin{aligned}
& u_{r}^{* f}=-Q_{3}^{* s} D_{1}^{*}\left(\varpi k^{s} k^{f} \Delta+\xi P_{1}^{*}\right) \frac{z r}{\varrho^{2}}\left(\frac{\partial^{2} E}{\partial \varrho^{2}}-\frac{1}{\varrho} \frac{\partial E}{\partial \varrho}\right) \\
& u_{\theta}^{* f}=0, \\
& u_{z}^{* f}=\Delta Q_{3}^{* s} D_{1}^{*}\left(\varpi k^{s} k^{f} \Delta+\xi P_{1}^{*}\right) E-Q_{3}^{* s} D_{1}^{*}\left(\varpi k^{s} k^{f} \Delta+\xi P_{1}^{*}\right) \frac{1}{\varrho^{2}}\left(\frac{\partial^{2} E}{\partial \varrho^{2}} z^{2}+\frac{r^{2}}{\varrho} \frac{\partial E}{\partial \varrho}\right), \\
& \text { and } \\
& \pi^{* f}=-i \omega \xi D_{1}^{*} D_{2}^{*} \frac{\partial E}{\partial z} .
\end{aligned}
$$

On the other hand, the microrotations are

$$
\begin{aligned}
& \phi^{* s}=-\Delta Q_{3}^{* s} D_{1}^{*}\left(k^{s} Q_{4}^{* f}-\mathrm{i} \omega \xi \varpi k^{f}\right) \operatorname{curl}(0,0, E), \\
& \phi^{* f}=\Delta Q_{3}^{* s} D_{1}^{*}\left(\varpi k^{s} Q_{1}^{* f}+\xi k^{f} Q_{2}^{* s}\right) \operatorname{curl}(0,0, E),
\end{aligned}
$$

and thus, we have

$$
\begin{aligned}
& \phi_{r}^{* s}=\phi_{z}^{* s}=0, \\
& \phi_{\theta}^{* s}=\Delta Q_{3}^{* s} D_{1}^{*}\left(k^{s} Q_{4}^{* f}-\mathrm{i} \omega \xi \varpi k^{f}\right) \frac{r}{\varrho} \frac{\partial E}{\partial \varrho}, \\
& \phi_{r}^{* f}=\phi_{z}^{* f}=0, \\
& \phi_{\theta}^{* f}=-\Delta Q_{3}^{* s} D_{1}^{*}\left(\varpi k^{s} Q_{1}^{* f}+\xi k^{f} Q_{2}^{* s}\right) \frac{r}{\varrho} \frac{\partial E}{\partial \varrho}
\end{aligned}
$$

and the solution of the point force problem is complete.

Let now consider that the concentrated couple $\mathbf{L}^{* s}(\mathbf{x})=\delta(\mathbf{x}-\mathbf{y}) \mathbf{m}$ is applied to the mixture. 
As above, we will find that the corresponding solution is

$$
\begin{aligned}
& u_{r}^{* s}=u_{z}^{* s}=0, \\
& u_{\theta}^{* s}=\Delta Q_{3}^{* s} D_{1}^{*}\left(k^{s} Q_{4}^{* f}-\mathrm{i} \omega \xi \varpi k^{f}\right) \frac{r}{\varrho} \frac{\partial E}{\partial \varrho}, \\
& \phi_{r}^{* s}=\Delta Q_{3}^{* s} D_{4}^{*} \frac{z r}{\varrho^{2}}\left(\frac{\partial^{2} E}{\partial \varrho^{2}}-\frac{1}{\varrho} \frac{\partial E}{\partial \varrho}\right), \\
& \phi_{\theta}^{* s}=0, \\
& \phi_{z}^{* s}=\Delta Q_{3}^{* s} D_{1}^{*}\left[\left(k^{f}\right)^{2} \Delta Q_{1}^{* s}-P_{2}^{*} Q_{2}^{* f}\right] E+\Delta Q_{3}^{* s} D_{4}^{*} \frac{1}{\varrho^{2}}\left(\frac{\partial^{2} E}{\partial \varrho^{2}} z^{2}+\frac{r^{2}}{\varrho} \frac{\partial E}{\partial \varrho}\right), \\
& u_{r}^{* f}=u_{z}^{* f}=0, \\
& u_{\theta}^{* f}=-\Delta Q_{3}^{* s} D_{1}^{*}\left(\varpi k^{f} Q_{1}^{* s}+\xi k^{s} Q_{2}^{* f}\right) \frac{r}{\varrho} \frac{\partial E}{\partial \varrho}, \\
& \phi_{r}^{* f}=\Delta Q_{3}^{* s} D_{5}^{*} \frac{z r}{\varrho^{2}}\left(\frac{\partial^{2} E}{\partial \varrho^{2}}-\frac{1}{\varrho} \frac{\partial E}{\partial \varrho}\right), \\
& \phi_{\theta}^{* s}=0, \\
& \phi_{z}^{* s}=\Delta Q_{3}^{* s} D_{1}^{*}\left(\xi k^{s} k^{f} \Delta+\varpi P_{2}^{*}\right) E-\Delta Q_{3}^{* s} D_{5}^{*} \frac{1}{\varrho^{2}}\left(\frac{\partial^{2} E}{\partial \varrho^{2}} z^{2}+\frac{r^{2}}{\varrho} \frac{\partial E}{\partial \varrho}\right), \\
& \pi^{* f}=0 .
\end{aligned}
$$

Similarly we can find the solutions which correspond to the concentrated loads $\mathbf{F}^{* f}(\mathbf{x})=\delta(\mathbf{x}-\mathbf{y}) \mathbf{m}$ and $\mathbf{L}^{* f}(\mathbf{x})=\delta(\mathbf{x}-\mathbf{y}) \mathbf{m}$ and the problem is solved.

\section{Acknowledgment}

This work was supported by CNCSIS -UEFISCSU, project number 15 PNII - IDEI code ID-401/2007. The author would like to express their gratitude to the referees for their careful assessment as well as for their fruitful suggestions regarding the initial version of the paper.

\section{References}

de Boer, R., Svanadze, M., 2004. Fundamental Solution of the System of Equations of Steady Oscillations in the Theory of Fluid-Saturated Porous Media. Transp. in Porous Media 56, 39-50.

de Boer, R., 2005. Trends in continuum mechanics of porous media. Springer, Berlin. 
Chiriţă, S., Galeş, C., 2008. A Mixture Theory for Microstretch Thermoviscoelastic Solids. Journal of Thermal Stresses 31, 1099-1124.

Ciarletta, M., 1995. General Theorems and Fundamental Solutions in the Dynamical Theory of Mixtures. J. Elasticity 39, 229-246.

Ciarletta, M., Svanadze, M., Buonannoa, L., 2009. Plane Waves and Vibrations in the Theory of Micropolar Thermoelasticity for Materials with Voids. Eur. J. Mech. A-Solids doi:10.1016/j.euromechsol.2009.03.008.

Dragoş, L., Homentcovschi, D., 1980. Stationary Fundamental Solution for an Ideal Fluid in Uniform Motion. ZAMM 60, 343-346.

Dragoş, L., Homentcovschi, D., 1983. Fundamental Matrices in Micropolar Fluids. ZAMM 63, 389-391.

Elangovan, S., Altan, B.S., Odegard, G.M., 2008. An Elastic Micropolar Mixture Theory for Predicting Elastic Properties of Cellular Materials. Mech. Mater. 40, 602-614.

Eringen, A.C., 1999. Microcontinuum field theories. Foundations and Solids. Springer, New York.

Eringen, A.C., 2001. Microcontinuum field theories, II, Fluent Media. Springer, New York.

Eringen, A.C., 2003. Micropolar Mixture Theory of Porous Media. J. Appl. Phys. 94, 4184-4190.

Gales, C., 2004-a. Waves and Vibrations in the Theory of Swelling Porous Elastic Solis. Eur. J. Mech. A-Solids 23, 345-357.

Galeş, C., 2004-b. Potential Method in the Linear Theory of Swelling Porous Elastic soils. Eur. J. Mech. A-Solids 23, 957-973.

Ghiba, I.D., 2006. Some Uniqueness and Continuous Dependence Results in the Micropolar Mixture Theory of Porous Media. Int. J. Engng. Sci. 44, 1269-1279.

Ghiba, I.D., 2007. Existence and Uniqueness Results in the Micropolar Mixture Theory of Porous Media, in Cârjă, O., Vrabie, I (Eds.), Applied analysis and differential equations. World Scientific, New Jersey, pp. 139-152.

Ghiba, I.D., 2008. Asymptotic Partition of Energy in Micropolar Mixture Theory Of Porous Media. Meccanica 43, 639-649. 
Ghiba, I.D., 2009. Some Uniqueness and Stability Results in the Theory of Micropolar Solid-Fluid Mixture. J. Math. Anal. Appl. 355, 385-396.

Hörmander, L., 1964. Linear partial differential operators. Springer, Berlin.

Ieşan, D., 1971. Existence Theorem in Micropolar Elastostatics. Int. J. Engng. Sci. 9, 59-78.

Ieşan, D., Pompei, A., 1995. On the Equilibrium Theory of Microstrech Elastic Solids. Int. J. Engng. Sci. 33, 399-410.

Ieşan, D., 1996. Existence Theorem in the Theory of Mixtures. J. Elasticity $42,145-163$.

Ieşan, D., Nappa, L., 2001. On the Plane Strain of Microstrech Elastic Solids. Int. J. Engng. Sci. 39, 1815-1835.

Ieşan, D., 2007. A Theory of Thermoviscoelastic Composites Modelled as Interacting Cosserat Continua. Journal of Thermal Stresses 30, 1269-1289.

Ieşan, D., 2009. Binary Mixtures of Elastic Solids with Microstructure. Mathematics and Mechanics of Solids 14, 564-586.

Kythe, P.K., 1996. Fundamental solutions for differential operators and applications, Birkhauser, Boston.

Lakes, R.S., 1983. Size Effects and Micromechanics of a Porous Solid. J. Mater. Sci. 18, 2572-2580.

Lakes, R.S., 1986. Experimental Microelasticity of Two Porous Solids. Int. J. Solids Structures 22, 55-63.

Moisil, Gr.C., 1952. Teoria preliminara a sistemelor de ecuatii cu derivate partiale liniare cu coeficienti constanti. Bul. Şt. Acad. R.P.R., Secţ. şt. Mat. Fiz. 4, 319-401.

Mora, R. \& Waas, A. M., 2000. Measurement of The Cosserat Constant of Circular-Cell Polycarbonate Foam. Phil. Mag. A 80, 1699-1713.

Nappa, L., 2008. Fundamental Solutions in the Theory of Microfluids. Ann. Univ. Ferrara 54, 95-106.

Olmstead, W.E., Majumdar, S.R., 1983. Fundamental Oseen Solution for the 2-dimensional Flow of a Micropolar Fluid. Int. J. Engng. Sci. 21, 423-430. 
Rajagopal, K.R., Tao, L., 1995. Mechanics of mixtures, Series on Advances in Mathematics for Applied Science vol. 35. World Scientific, Singapore.

Ramkissoon, H., Majundar, S.R., 1976-a. Drag on an Axially Symmetric Body in the Stokes' Flow of Micropolar Fluid. Phys. Fluids 19, 16-21.

Ramkissoon, H., Majundar, S.R., 1976-b. Representations and Fundamental Singular Solutions in Micropolar Fluid. ZAMM 56, 197-203.

Shu, J.-J., Lee, J.S., 2008. Fundamental Solutions for Micropolar Fluids. J. Eng. Math. 61, 69-79.

Svanadze, M., 1993. Representation of the General Solution of the Equation of Steady Oscillations of Two-component Elastic Mixture. Int. Appl. Mech. 29, 22-29.

Svanadze, M., 1996. The Fundamental Solution of the Oscillation Equations of the Thermoelasticity Theory of Mixture of Two Elastic Solids. J. Thermal Stresses 19, 633-648.

Svanadze, M., de Boer, R., 2005. Representations of Solutions in the Theory of Fluid-Saturated Porous Media. Quart. J. Mech. Appl. Math. 58, 551562 .

Svanadze, M., Giorano, P., Zampoli, V., 2007. On Representations of a General Solution in the Theory of Micropolar Thermoelasticity without Energy Dissipation. Ukrainian Mathematical Journal 59, 1590-1568.

Sandru, N., 1966. On Some Problems of the Linear Theory of the Asymmetric Elasticity. Int. J. Engng. Sci. 4, 81-94.

Sandru, N., 1975. The Relation Between the Fundamental Solution in E3 and that in E2 Micropolar Elasticity. Mech. Res. Comm. 2, 27-30.

Tekoglu, C., Onck, P.R., 2008. Size Effects in Two-Dimensional Voronoi Foams: A Comparison Between Generalized Continua and Discrete Models. J. Mech. Phys. Solids 56, 3541-3564.

Singh, D., Tomar, S.K., 2006. Wave Propagation in Micropolar Mixture of Porous Media. Int. J. Engng. Sci. 44 , 1304-1323.

Twiss, R.J., Eringen, A.C., 1971. Theory of Mixtures for Micromorphic Materials I. Int. J. Engng. Sci. 9, 1019-1044.

Twiss, R.J., Eringen, A.C., 1972. Theory of Mixtures for Micromorphic Materials II., Elastic Constitutive Equations. Int. J. Engng. Sci. 10, 437-465. 